\title{
Crible étrange et sommes de Kloosterman
}

\author{
par \\ Jimena SivaK-Fischler (Orsay)
}

1. Introduction. On réserve la lettre $p$ aux nombres premiers.

On rappelle que la somme de Kloosterman $\operatorname{Kl}(a, b ; n)$ est définie, pour $a, b, n \geq 1$ entiers, par la formule

$$
\mathrm{Kl}(a, b ; n)=\sum_{\substack{x \bmod n \\(x, n)=1}} \exp \left(2 i \pi \frac{a x+b \bar{x}}{n}\right)
$$

où $(x, n)$ est le pgcd de $x$ et $n$, et $\bar{x}$ l'inverse de $x$ modulo $n$. Ce sont des nombres réels, non nuls pour $n=p$ premier.

Pour $(m, n)=1$, les sommes de Kloosterman vérifient la multiplicativité croisée $([6$, p. 19])

$$
\mathrm{Kl}(a, b ; m n)=\operatorname{Kl}(a \bar{m}, b \bar{m} ; n) \operatorname{Kl}(a \bar{n}, b \bar{n} ; m) .
$$

On a la majoration suivante (cf. [1, formule (6)]) pour $n \geq 1$ :

$$
|\mathrm{Kl}(a, b ; n)| \leq \tau(n)(a, b, n)^{1 / 2} n^{1 / 2},
$$

où $\tau(n)$ désigne le nombre de diviseurs de $n$. Cette majoration est fréquemment appelée majoration d'Estermann-Weil.

Pour $n \geq 1$ on définit

$$
\mathrm{Kl}^{*}(1,1 ; n):=\frac{\mathrm{Kl}(1,1 ; n)}{\sqrt{n}}
$$

et l'angle $\theta_{p, a}$ de la somme de Kloosterman par

$$
\cos \theta_{p, a}=\frac{\mathrm{Kl}(1, a ; p)}{2 \sqrt{p}} \quad \text { avec } 0 \leq \theta_{p, a} \leq \pi .
$$

Cet angle est bien défini puisqu'on a vu que $|\operatorname{Kl}(1, a ; p)| \leq 2 \sqrt{p}$.

Un premier résultat, dû à Kuznetsov (cf. [7, théorème 3]), montre qu'il existe de nombreuses compensations entre ces nombres :

2000 Mathematics Subject Classification: Primary 11N36; Secondary 11L05, 11N35. 


$$
\sum_{n} g(n / X) \mathrm{Kl}^{*}(1,1 ; n)=\mathrm{o}_{g}(X),
$$

pour toute fonction $g \in C^{\infty}(\mathbb{R}, \mathbb{R})$ à support inclus dans $[1,2]$.

Soit $\mu_{\mathrm{ST}}$ la mesure de Sato-Tate $2 \pi^{-1} \sin ^{2} \theta d \theta$ sur $[0, \pi]$. Katz a démontré la loi de Sato-Tate verticale ([6, p. 492]) :

ThÉORÈme 1.1 (Katz). L'ensemble des angles $\left\{\theta_{p, a} \mid 1 \leq a \leq p\right\}$ est équiréparti sur $[0, \pi]$, pour $p \rightarrow+\infty$, suivant $\mu_{\mathrm{ST}}$.

Katz a ensuite conjecturé la loi de Sato-Tate horizontale ([6, p. 493]) :

Conjecture 1.2 (Katz). Pour $p \rightarrow+\infty$, l'ensemble des angles $\theta_{p, 1}$ est équiréparti sur $[0, \pi]$ suivant $\mu_{\mathrm{ST}}$.

Pour l'instant, cette conjecture ne semble pas accessible. Elle amène cependant à considérer un problème plus simple : savoir si les sommes de Kloosterman $\mathrm{Kl}(1,1 ; p)$ changent une infinité de fois de signe. Ce problème bien plus simple semble aussi hors d'atteinte pour l'instant.

Le seul résultat connu dans cette direction est dû à Fouvry et Michel ([2, théorème 2]) :

THÉORÈme 1.3. Il existe $X_{0}>0$ et $c_{0}>0$ tels que pour $X \geq X_{0}$ et pour $z=X^{1 / 23.9}$, on ait les minorations

$$
\begin{aligned}
& \left|\left\{n \in[X, 2 X]\left|\mathrm{Kl}^{*}(1,1 ; n)>0, p\right| n \Rightarrow p \geq z\right\}\right| \geq c_{0} \frac{X}{\log X}, \\
& \left|\left\{n \in[X, 2 X]\left|\mathrm{Kl}^{*}(1,1 ; n)<0, p\right| n \Rightarrow p \geq z\right\}\right| \geq c_{0} \frac{X}{\log X} .
\end{aligned}
$$

Pour aller plus loin, Fouvry et Michel émettent la conjecture suivante pour tout $0<\theta \leq 1$ :

Conjecture $1.4(H(\theta))$. Pour toute fonction $g \in C^{\infty}(\mathbb{R}, \mathbb{R})$ à support inclus dans $[1,2]$, pour tout $\varepsilon>0$, tout $A \geq 0$ et tout $X \geq 2$, on a

$$
\sum_{d \leq X^{\theta-\varepsilon}}\left|\sum_{d \mid n} g(n / X) \mathrm{Kl}^{*}(1,1 ; n)\right|=\mathrm{O}_{g, \varepsilon, A}\left(X \log ^{-A} X\right) .
$$

En admettant $H(1)$, Fouvry et Michel ont démontré dans [3] l'existence d'une infinité de changements de signe des sommes de Kloosterman $\mathrm{Kl}(1,1 ; n)$ pour $n$ ayant au plus 3 facteurs premiers (en utilisant le crible asymptotique). Pour l'instant, cependant, la conjecture 1.4 semble hors d'atteinte. Fouvry et Michel la démontrent pour $\theta \leq 1 / 2$ (proposition 2.1 de [2] et proposition 3.3 ci-dessous). En utilisant ce résultat inconditionnel à la place de $H(1)$, ils n'obtiennent (voir [3]) que 23 à la place de 3, ce qui est moins fort que le théorème 1.3 puisque la taille des facteurs premiers de $n$ n'est pas précisée. 
On peut maintenant énoncer le théorème principal de cet article :

ThéORÈme 1.5. Il existe $X_{0}>0$ et $c_{0}>0$ tels que pour $X \geq X_{0}$ et pour $z=X^{1 / 22.29}$, on ait les minorations

$$
\begin{aligned}
& \left|\left\{n \in[X, 2 X]\left|\mathrm{Kl}^{*}(1,1 ; n)>0, p\right| n \Rightarrow p \geq z\right\}\right| \geq c_{0} \frac{X}{\log X}, \\
& \left|\left\{n \in[X, 2 X]\left|\mathrm{Kl}^{*}(1,1 ; n)<0, p\right| n \Rightarrow p \geq z\right\}\right| \geq c_{0} \frac{X}{\log X} .
\end{aligned}
$$

On a donc démontré qu'une proportion positive d'entiers $n$ (avec tous leurs facteurs premiers $\geq n^{1 / 22.29}$ ) sont tels que $\mathrm{Kl}(1,1 ; n)>0$. On a également démontré le même résultat pour $\mathrm{Kl}(1,1 ; n)<0$. Il y a donc une infinité de changements de signe des sommes de Kloosterman sur l'ensemble des $n$ dont tous les facteurs premiers sont $\geq n^{1 / 22.29}$. Ceci améliore le théorème 1.3, puisqu'on a $1 / 22.29$ à la place de $1 / 23.9$.

On déduit du théorème 1.5 qu'il existe une infinité de changements de signe des sommes de Kloosterman sur l'ensemble des $n$ ayant au plus 22 facteurs premiers. On peut en fait remplacer 22 par 18 grâce aux résultats de [8] (mais la preuve utilise des méthodes différentes : le crible asymptotique de Bombieri et la loi de Sato-Tate verticale entre autres). Cependant, le théorème 1.5 lui-même est plus précis, car les facteurs premiers de $n$ doivent être grands.

Pour démontrer le théorème 1.3, Fouvry et Michel ont introduit le crible étrange, qui est une version modifiée du crible de Selberg. Cette modification consiste à remplacer l'hypothèse habituelle du crible de Selberg (cf. [5, p. 28])

$$
\sum_{d \mid n} a_{n}=\frac{\omega(d)}{d} X+r_{d}
$$

où $\omega$ est une fonction multiplicative, $X \geq 2$ et $r_{d}$ est un terme d'erreur, par l'hypothèse du crible étrange

$$
\sum_{d \mid n} a_{n}=\frac{\omega(d)}{d} Y-\frac{\omega(d)}{d}(\log d) Z+r_{d}
$$

avec $Y, Z \geq 2$. On peut ainsi voir le terme en $Z$ comme un terme correctif.

Pour démontrer le théorème 1.5, on reprend le crible étrange (§2), mais on l'itère une fois grâce à l'identité de Buchstab. Alors que Fouvry et Michel disposaient seulement d'une majoration de $S(\mathcal{A}, z):=\sum_{n, p \mid n \Rightarrow p \geq z} a_{n}$, on obtient aussi une minoration qui est de la forme suivante (théorème 2.8 ci-dessous) : 


$$
\begin{aligned}
S(\mathcal{A}, z) \geq & W(z)\left\{Y\left(1-\eta_{2}(2 \tau)\right)+Z h_{1}(2 \tau) \log x+Z\left(2+h_{2}(2 \tau)\right) \log z\right. \\
& \left.+\mathrm{O}\left(\left(Y+Z \frac{\log ^{2} z}{\log x}\right) \frac{\log _{2}^{8} x}{\log x}\right)\right\}-\sum_{\substack{d \mid P(z) \\
d \leq x^{2}}} 3^{\Omega(d)}\left|r_{d}\right|,
\end{aligned}
$$

avec $\tau=\log x / \log z$ et $\eta_{2}, h_{1}$ et $h_{2}$ des fonctions de $] 1,+\infty[$ dans $\mathbb{R}$. Cette minoration est à rapprocher de celle qui apparaît dans le crible de Selberg habituel (cf. [5, p. 215]) :

$$
S(\mathcal{A}, z) \geq X W(z)\left\{1-\eta_{2}(2 \tau)+\mathrm{O}\left(\frac{\log _{2}^{8} x}{\log x}\right)\right\}-\sum_{\substack{d \mid P(z) \\ d \leq x^{2}}} 3^{\Omega(d)}\left|r_{d}\right|
$$

La différence provient de termes correctifs en $Z$, introduits dans l'hypothèse $(2)$.

Comme Fouvry et Michel, on utilise des résultats issus de la théorie des formes automorphes (à travers $H(1 / 2)$, §3.2) pour majorer

$$
\left|\sum_{p \mid n \Rightarrow p \geq X^{1 / v}} g(n / X) \mathrm{Kl}^{*}(1,1 ; n)\right|,
$$

et de géométrie algébrique (§3.4) pour minorer

$$
\sum_{p \mid n \Rightarrow p \geq X^{1 / v}}\left|g(n / X) \mathrm{Kl}^{*}(1,1 ; n)\right| .
$$

Une différence majeure avec la preuve de Fouvry et Michel est l'apparition d'intégrales du type $\eta_{2}$ sur des intervalles non bornés, en la fonction classique $\sigma_{2}$ qui est définie par une équation différentielle aux différences. Il est donc nécessaire de tronquer l'intervalle en contrôlant le reste (§3.3), avant de pouvoir calculer l'intégrale sur un intervalle fini par la méthode des rectangles.

Remerciements. Je tiens à remercier chaleureusement mon directeur de thèse E. Fouvry pour m'avoir proposé de travailler sur ce sujet, ainsi que $\mathrm{Ph}$. Michel pour ses conseils.

2. Le crible étrange. Dans cette partie, on rappelle les notations et hypothèses du crible étrange $(\S 2.1)$, puis la majoration obtenue par Fouvry et Michel (\$2.2). On en déduit (\$2.3) la majoration asymptotique dont nous aurons besoin dans la suite. On démontre au $\$ 2.4$ le lemme fondamental du crible étrange, ce qui nous permet (\$2.5) d'obtenir une minoration de $S(\mathcal{A}, z)$.

2.1. Notations et définitions. Introduisons d'abord quelques notations, et l'hypothèse du crible étrange. 
Soit $X>0$ et soit $\mathcal{A}=\mathcal{A}(X)$ une suite de réels $a_{n}=a_{n}(X)$ tels que

$$
\begin{cases}a_{n} \geq 0 & \text { pour } n \geq 1 \\ a_{n}=0 & \text { pour } n \notin[X, 2 X] .\end{cases}
$$

Pour $z \geq 2$, on pose

$$
S(\mathcal{A}, z):=\sum_{n, p \mid n \Rightarrow p \geq z} a_{n}
$$

Pour tout entier $d \geq 1$, on pose

$$
\left|\mathcal{A}_{d}\right|:=\sum_{d \mid n} a_{n}
$$

et l'on a $|\mathcal{A}|:=\sum_{n} a_{n}=\left|\mathcal{A}_{1}\right|$. On suppose que cette quantité peut s'écrire pour $d$ sans facteur carré sous la forme

$$
\left|\mathcal{A}_{d}\right|=\frac{\omega(d)}{d} Y-\frac{\omega(d)}{d}(\log d) Z+r_{d}
$$

où $Y$ et $Z$ sont des réels positifs indépendants de $d$, et $\omega$ est la fonction multiplicative telle que

$$
\omega(2)=0, \quad \omega(p)=2 \quad \text { pour } p>2 .
$$

Remarquons que pour $Z=0$, on reconnaît l'hypothèse classique du crible de Selberg. En outre, nous nous restreignons volontairement à un cas particulier de crible étrange de dimension 2 et n'envisageons pas le cas général où $\omega(p)$ vaut 2 en moyenne.

2.2. Premières majorations de $S(\mathcal{A}, z)$. On rappelle tout d'abord la proposition 3.1 de [2] :

Proposition 2.1. Sous les hypothèses (3), (4) et avec $\omega$ fonction multiplicative telle que $0 \leq \omega(p)<p$ pour tout $p$ premier, on a pour tout $2 \leq z \leq x$ la majoration

où

$$
\begin{aligned}
S(\mathcal{A}, z) \leq & \frac{Y}{G(x, z)}+\frac{Z}{G(x, z)^{2}} \sum_{p<z} \frac{\omega(p)}{p} \frac{\log p}{(1-\omega(p) / p)^{2}} G_{p}(x / p, z) \\
& +\sum_{\substack{d \mid P(z) \\
d \leq x^{2}}} 3^{\Omega(d)}\left|r_{d}\right|
\end{aligned}
$$

$$
\begin{gathered}
P(z):=\prod_{p<z} p, \quad G(x, z):=\sum_{\substack{d \mid P(z) \\
d \leq x}} \frac{\omega(d)}{d \prod_{p \mid d}(1-\omega(p) / p)}, \\
G_{q}(y, z):=\sum_{\substack{d \mid P(z),(q, d)=1 \\
d \leq y}} \frac{\omega(d)}{d \prod_{p \mid d}(1-\omega(p) / p)},
\end{gathered}
$$

et $\Omega(d)$ est le nombre de facteurs premiers de d, comptés avec multiplicité. 
Dans la suite, on utilisera cette proposition à travers le corollaire suivant, dont nous aurons besoin après avoir appliqué l'identité de Buchstab :

Corollaire 2.2. Sous les hypothèses (3)-(5), pour tout $2 \leq z \leq x$ et pour tout entier $q$ sans facteur carré tel que $(q, P(z))=1$ et $Y-Z \log q \geq 0$, on a l'inégalité

$$
\begin{aligned}
& S\left(\mathcal{A}_{q}, z\right) \\
\leq & \frac{\omega(q)}{q} \frac{Y}{G(x, z)} \\
& +\frac{\omega(q)}{q} \frac{Z}{G(x, z)}\left\{\frac{1}{G(x, z)} \sum_{p<z} \frac{\omega(p)}{p} \frac{\log p}{(1-\omega(p) / p)^{2}} G_{p}(x / p, z)-\log q\right\} \\
& +\sum_{\substack{d \mid P(z) \\
d \leq x^{2}}} 3^{\Omega(d)}\left|r_{d q}\right| .
\end{aligned}
$$

Preuve. Comme $\omega$ est multiplicative, l'égalité (4) donne, pour $d$ sans facteur carré tel que $(d, q)=1$,

$$
\left|\mathcal{A}_{d q}\right|=\frac{\omega(d)}{d} \frac{\omega(q)}{q}(Y-(\log q) Z)-\left(\frac{\omega(d)}{d} \log d\right) \frac{\omega(q)}{q} Z+r_{d q} .
$$

Soit $\left(\lambda_{d}\right)_{d \geq 1}$ une suite de réels vérifiant

$$
\lambda_{1}=1, \quad \lambda_{d}=0 \quad \text { si } d \nmid P(z) \text { ou } d>x .
$$

On a alors l'inégalité

$$
S\left(\mathcal{A}_{q}, z\right) \leq \sum_{n} a_{q n}\left(\sum_{d \mid(n, P(z))} \lambda_{d}\right)^{2} \leq \sum_{d_{1} \mid P(z)} \sum_{d_{2} \mid P(z)} \lambda_{d_{1}} \lambda_{d_{2}}\left|\mathcal{A}_{q\left[d_{1}, d_{2}\right]}\right| .
$$

On a alors, par (7),

avec

$$
S\left(\mathcal{A}_{q}, z\right) \leq \frac{\omega(q)}{q}\left(Y Q_{1}-Z Q_{2}\right)+R_{q}
$$

$$
\begin{aligned}
Q_{1} & :=\sum_{d_{1} \mid P(z)} \sum_{d_{2} \mid P(z)} \frac{\omega\left(\left[d_{1}, d_{2}\right]\right)}{\left[d_{1}, d_{2}\right]} \lambda_{d_{1}} \lambda_{d_{2}}, \\
Q_{2} & :=\sum_{d_{1} \mid P(z)} \sum_{d_{2} \mid P(z)} \frac{\omega\left(\left[d_{1}, d_{2}\right]\right)}{\left[d_{1}, d_{2}\right]} \log \left(\left[d_{1}, d_{2}\right]\right) \lambda_{d_{1}} \lambda_{d_{2}}, \\
R_{q} & :=\sum_{d_{1} \mid P(z)} \sum_{d_{2} \mid P(z)} \lambda_{d_{1}} \lambda_{d_{2}} r_{q\left[d_{1}, d_{2}\right]} .
\end{aligned}
$$

On minimise la forme quadratique $Q_{1}$ par un choix optimal de la suite $\left(\lambda_{d}\right)_{d \geq 1}$, comme dans la preuve de la proposition 3.1 de [2], et l'on trouve 
pour ce choix particulier de $\left(\lambda_{d}\right)_{d \geq 1}$ que

$$
Q_{1}=\frac{1}{G(x, z)}
$$

avec $\left|\lambda_{d}\right| \leq 1$ (équation (3.7) dans la preuve de la proposition 3.1 de [2]). Ceci entraîne

$$
\left|R_{q}\right| \leq \sum_{\substack{d_{1}, d_{2} \mid P(z) \\ d_{1}, d_{2} \leq x}}\left|r_{q\left[d_{1}, d_{2}\right]}\right| \leq \sum_{\substack{d \mid P(z) \\ d \leq x^{2}}} 3^{\Omega(d)}\left|r_{d q}\right|
$$

car $\lambda_{d}=0$ pour $d>x$, par définition. Enfin, on a

$$
Q_{2}=-\frac{1}{G(x, z)^{2}} \sum_{p<z} \frac{\omega(p)}{p} \frac{\log p}{(1-\omega(p) / p)^{2}} G_{p}(x / p, z)
$$

(voir l'équation (3.10) de [2]), ce qui termine la preuve du corollaire 2.2.

REMARque. On peut mettre en parallèle (6) avec la majoration classique dans le cas du crible de Selberg (cf. [5, p. 191])

$$
S\left(\mathcal{A}_{q}, z\right) \leq \frac{\omega(q)}{q} \frac{X}{G(x, z)}+\sum_{\substack{d \mid P(z) \\ d \leq x^{2}}} 3^{\Omega(d)}\left|r_{d q}\right| .
$$

2.3. Majoration asymptotique de $S(\mathcal{A}, z)$. On déduit du corollaire 2.2 la proposition suivante, qui n'est valable que pour $z$ assez grand par rapport à $x$ :

Proposition 2.3. Sous les hypothèses (3)-(5), on a la majoration suivante, uniformément pour tout $\exp \left((\log x)^{6 / 7}\right) \leq z \leq \sqrt{x}$ et pour tout entier $q$ sans facteur carré tel que $(q, P(z))=1$ et $Y-Z \log q \geq 0$ :

(8) $S\left(\mathcal{A}_{q}, z\right)$

$$
\begin{aligned}
\leq & \frac{\omega(q)}{q} \frac{W(z)}{\sigma_{2}(2 \tau)}\{Y-Z \log q \\
& +2 Z(\log z)\left\{\frac{\tau}{2}+\frac{1+\tau}{4} \frac{\sigma_{2}(2 \tau+2)}{\sigma_{2}(2 \tau)}+\frac{3(1-\tau)}{4} \frac{\sigma_{2}(2 \tau+4)}{\sigma_{2}(2 \tau)}\right\} \\
& \left.+\mathrm{O}\left(\frac{\tau^{5}}{\log z}(Y-Z \log q+2 Z \log z)\right)\right\}+\sum_{\substack{d \mid P(z) \\
d \leq x^{2}}} 3^{\Omega(d)}\left|r_{d q}\right|
\end{aligned}
$$

avec

$$
\tau:=\frac{\log x}{\log z}, \quad W(z):=\prod_{2<p<z}\left(1-\frac{2}{p}\right),
$$

et la fonction $\sigma_{2}: \mathbb{R}^{+} \rightarrow \mathbb{R}$ continue définie par l'équation différentielle aux 
différences

$$
\begin{cases}\sigma_{2}(u)=u^{2} / 8 e^{2 \gamma} & \text { pour } 0 \leq u \leq 2 \\ \left(u^{-2} \sigma_{2}(u)\right)^{\prime}=-2 u^{-3} \sigma_{2}(u-2) & \text { pour } u \geq 2\end{cases}
$$

Preuve. On commence par suivre la preuve du corollaire 3.2 de [2] en écrivant, pour $2<p<z \leq \sqrt{x}$, les relations (d'après [5, p. 197])

$$
\frac{1}{G(x, z)}=W(z)\left\{\frac{1}{\sigma_{2}(2 \tau)}+\mathrm{O}\left(\frac{\tau^{5}}{\log z}\right)\right\}
$$

et

$$
\frac{1}{G_{p}(x / p, z)}=W(z)\left(1-\frac{2}{p}\right)^{-1}\left\{\frac{1}{\sigma_{2}(2 \tau-2 \log p / \log z)}+\mathrm{O}\left(\frac{\tau^{5}}{\log z}\right)\right\},
$$

qui entraînent l'égalité

$$
G_{p}(x / p, z)=W(z)^{-1}\left(1-\frac{2}{p}\right)\left\{\sigma_{2}\left(2 \tau-2 \frac{\log p}{\log z}\right)+\mathrm{O}\left(\frac{\tau^{5}}{\log z}\right)\right\}
$$

car $\tau^{5} / \log z \rightarrow 0$ quand $x \rightarrow+\infty$ par hypothèse (ceci n'est vrai que pour $z$ suffisamment grand, d'où l'hypothèse $\left.z \geq \exp \left((\log x)^{6 / 7}\right)\right)$.

Ainsi, en faisant une sommation par parties et en appliquant le théorème des nombres premiers, comme dans [2] on obtient

$$
\begin{aligned}
\sum_{p<z} \frac{\omega(p)}{p} \frac{\log p}{(1-\omega(p) / p)^{2}} & G_{p}(x / p, z) \\
& =2 W(z)^{-1}(\log z) \int_{0}^{1} \sigma_{2}(2 \tau-2 t) d t+\mathrm{O}\left(\tau^{5}\right) .
\end{aligned}
$$

Alors que Fouvry et Michel conservent cette intégrale, on la majore pour éviter l'apparition d'une intégrale double lors de l'itération du crible et éviter ainsi un calcul d'erreur sur des intégrales doubles. Toutefois, la perte de précision dans la majoration est minuscule dans notre application. On utilisera la majoration du corollaire 2.5 ci-dessous, que nous démontrons en se servant d'un lemme :

Lemme 2.4. Pour $\tau \geq 1$, on a l'égalité

$$
\int_{0}^{1} \sigma_{2}(2 \tau-2 t) d t=\frac{\tau}{2} \sigma_{2}(2 \tau)-\frac{1+\tau}{2} \sigma_{2}(2 \tau+2)+\frac{3}{2} \int_{0}^{1} \sigma_{2}(2(\tau+1)-2 t) d t .
$$

Preuve. On pose $2 \tau-2 t=u-2$, donc l'intégrale devient

$$
\int_{0}^{1} \sigma_{2}(2 \tau-2 t) d t=\frac{1}{2} \int_{2 \tau}^{2 \tau+2} \sigma_{2}(u-2) d u .
$$


Or, en intégrant par parties, on a

$$
\int_{2 \tau}^{2 \tau+2} \sigma_{2}(u-2) d u=-\frac{1}{2}\left((2 \tau+2) \sigma_{2}(2 \tau+2)-2 \tau \sigma_{2}(2 \tau)\right)+\frac{3}{2} \int_{2 \tau}^{2 \tau+2} \sigma_{2}(u) d u .
$$

En faisant le changement de variable $u=2 \tau-2 t$, on a

$$
\int_{2 \tau}^{2 \tau+2} \sigma_{2}(u) d u=2 \int_{-1}^{0} \sigma_{2}(2 \tau-2 t) d t=2 \int_{0}^{1} \sigma_{2}(2(\tau+1)-2 t) d t
$$

ce qui entraîne le lemme.

On peut maintenant énoncer un corollaire immédiat de ce lemme, qui va nous permettre de majorer notre intégrale et de conclure la preuve de la proposition 2.3 :

Corollaire 2.5. Pour $\tau \geq 1$, on a la majoration

$$
\frac{1}{\sigma_{2}(2 \tau)} \int_{0}^{1} \sigma_{2}(2 \tau-2 t) d t \leq \frac{\tau}{2}+\frac{1+\tau}{4} \frac{\sigma_{2}(2 \tau+2)}{\sigma_{2}(2 \tau)}+\frac{3}{4}(1-\tau) \frac{\sigma_{2}(2 \tau+4)}{\sigma_{2}(2 \tau)} .
$$

Preuve. D'après le lemme 2.4, on a

$$
\begin{aligned}
& \frac{1}{\sigma_{2}(2 \tau)} \int_{0}^{1} \sigma_{2}(2 \tau-2 t) d t \\
& \quad=\frac{\tau}{2}-\frac{1+\tau}{2} \frac{\sigma_{2}(2 \tau+2)}{\sigma_{2}(2 \tau)}+\frac{3}{2} \frac{1}{\sigma_{2}(2 \tau)} \int_{0}^{1} \sigma_{2}(2(\tau+1)-2 t) d t .
\end{aligned}
$$

En appliquant à nouveau ce lemme (avec $\tau$ remplacé par $\tau+1$ ) à la dernière intégrale, on obtient

$$
\begin{aligned}
\frac{1}{\sigma_{2}(2 \tau)} \int_{0}^{1} \sigma_{2}(2 \tau- & 2 t) d t \\
= & \frac{\tau}{2}-\frac{1+\tau}{2} \frac{\sigma_{2}(2 \tau+2)}{\sigma_{2}(2 \tau)}+\frac{3}{2} \frac{1}{\sigma_{2}(2 \tau)}\left[\frac{\tau+1}{2} \sigma_{2}(2 \tau+2)\right. \\
& \left.-\frac{2+\tau}{2} \sigma_{2}(2 \tau+4)+\frac{3}{2} \int_{0}^{1} \sigma_{2}(2(\tau+2)-2 t) d t\right] .
\end{aligned}
$$

Comme $\sigma_{2}$ est croissante, on majore trivialement la dernière intégrale par

$$
\int_{0}^{1} \sigma_{2}(2(\tau+2)-2 t) d t \leq \sigma_{2}(2 \tau+4)
$$

d'où le corollaire.

Pour conclure la preuve de la proposition 2.3, il suffit d'appliquer le corollaire 2.2 , les relations (9) et (10), et le corollaire 2.5 . 
Remarque. Dans la preuve du corollaire 2.5, on aurait pu, au lieu de majorer trivialement la dernière intégrale, continuer à utiliser le lemme 2.4. Cela conduit à un raffinement du corollaire 2.5, mais n'apporte rien de significatif dans notre travail et complique les calculs.

2.4. Lemme fondamental du crible étrange. Dans ce paragraphe, on démontre le lemme fondamental du crible étrange (proposition 2.7). On entend par lemme fondamental un énoncé donnant un équivalent de $S(\mathcal{A}, z)$ pour $z$ beaucoup plus petit que $x$. Enonçons d'abord un lemme qui va nous servir dans la preuve (comme dans le cas du crible de Selberg) :

Lemme 2.6. Sous les hypothèses (3)-(5), pour tout $2 \leq z \leq x$ et pour tout entier $q$ sans facteur carré tel que $(q, P(z))=1$ et $Y-Z \log q \geq 0$, on a

$$
\begin{aligned}
S\left(\mathcal{A}_{q}, z\right) \leq & \frac{\omega(q)}{q} W(z)(1+\mathrm{O}(\exp (-\tau(\log \tau+2)))) \\
& \times\left\{Y-Z \log q+Z \sum_{p<z} \frac{\omega(p)}{p} \frac{\log p}{1-\omega(p) / p}\right\}+\sum_{\substack{d \mid P(z) \\
d \leq x^{2}}} 3^{\Omega(d)}\left|r_{d q}\right| .
\end{aligned}
$$

Preuve. Si $\tau>z$, on a

$$
\log x>z \log z>\sum_{p<z} \log p=\log P(z)
$$

donc

$$
G(x, z)=\sum_{d \mid P(z)} \frac{\omega(d)}{d \prod_{p \mid d}(1-\omega(p) / p)}=\frac{1}{W(z)} .
$$

Dans le cas contraire, c'est-à-dire si $\tau \leq z$, par le lemme 4.1 (p. 131) et la preuve du théorème 6.2 (p. 193) de [5] on a

$$
\frac{1}{G(x, z)} \leq W(z)(1+\mathrm{O}(\exp (-\tau(\log \tau+2)))) .
$$

Finalement, la majoration (11) est donc vérifiée dans les deux cas, c'est-à-dire pour tout $2 \leq z \leq x$.

D'autre part, pour $p<z$, en séparant les $d$ multiples de $p$ et ceux premiers à $p$, on a :

$$
G(x, z)=\sum_{\substack{d|P(z) \\ d \leq x \\ p| d}} \frac{\omega(d)}{d \prod_{p^{\prime} \mid d}\left(1-\omega\left(p^{\prime}\right) / p^{\prime}\right)}+\sum_{\substack{d \mid P(z) \\ d \leq x \\(d, p)=1}} \frac{\omega(d)}{d \prod_{p^{\prime} \mid d}\left(1-\omega\left(p^{\prime}\right) / p^{\prime}\right)}
$$




$$
\begin{aligned}
& =\left(\sum_{\substack{d \mid P(z) \\
d \leq x / p \\
(d, p)=1}} \frac{\omega(d)}{d \prod_{p^{\prime} \mid d}\left(1-\omega\left(p^{\prime}\right) / p^{\prime}\right)}\right) \frac{\omega(p)}{p(1-\omega(p) / p)} \\
& +\sum_{\substack{d \mid P(z) \\
d \leq x \\
(d, p)=1}} \frac{\omega(d)}{d \prod_{p^{\prime} \mid d}\left(1-\omega\left(p^{\prime}\right) / p^{\prime}\right)} \\
& \geq\left(\sum_{\substack{d \mid P(z) \\
d \leq x / p \\
(d, p)=1}} \frac{\omega(d)}{d \prod_{p^{\prime} \mid d}\left(1-\omega\left(p^{\prime}\right) / p^{\prime}\right)}\right)\left(1+\frac{\omega(p)}{p(1-\omega(p) / p)}\right) \\
& \geq G_{p}(x / p, z)\left(1-\frac{\omega(p)}{p}\right)^{-1} .
\end{aligned}
$$

Finalement, on a donc

$$
\frac{G_{p}(x / p, z)}{G(x, z)} \leq 1-\frac{\omega(p)}{p}
$$

En appliquant le corollaire 2.2 et en utilisant (11), on conclut la preuve du lemme 2.6.

La proposition suivante (lemme fondamental du crible étrange) donnera (§2.5) une minoration de $S(\mathcal{A}, z)$.

Proposition 2.7. Sous les hypothèses (3)-(5), on a, uniformément pour tout $5 \leq z \leq x^{1 / 4}$ tel que $Y-Z \log z \geq 0$,

$$
\begin{aligned}
S(\mathcal{A}, z)= & W(z)\{1+\mathrm{O}(\exp (-\tau(\log \tau+1)))\} \\
& \times\{Y+2 Z(\log z)(1+\mathrm{O}(1 / \log z))\}+\theta \sum_{\substack{d \mid P(z) \\
d \leq x^{2}}} 3^{\Omega(d)}\left|r_{d}\right|,
\end{aligned}
$$

avec un réel $\theta$ tel que $|\theta| \leq 1$.

REMARQUE. On peut comparer ce lemme fondamental (12) à celui du crible de Selberg (cf. [5, p. 206])

$$
S(\mathcal{A}, z)=X W(z)\{1+\mathrm{O}(\exp (-\tau(\log \tau+1)))\}+\theta \sum_{\substack{d \mid P(z) \\ d \leq x^{2}}} 3^{\Omega(d)}\left|r_{d}\right|
$$

Comme tout lemme fondamental, (12) est sans valeur pour $\tau$ borné, et est un équivalent quand $\tau \rightarrow+\infty$.

Preuve de la proposition 2.\%. Par l'identité de Buchstab (lemme 7.1, p. 204 de [5]) appliquée à $S(\mathcal{A}, z)$ et $W(z)$ on a 
(13)

$$
\begin{aligned}
S(\mathcal{A}, z) & -W(z) Y \\
& =S(\mathcal{A}, 5)-W(5) Y-\sum_{5 \leq p<z}\left\{S\left(\mathcal{A}_{p}, p\right)-\frac{\omega(p)}{p} W(p) Y\right\} .
\end{aligned}
$$

On l'utilise avec 5 (au lieu de l'utiliser avec 2 comme dans le cas du crible de Selberg) car $S(\mathcal{A}, 2)$ et $S(\mathcal{A}, 3)$ ne dépendent pas de $Z$, contrairement à $S(\mathcal{A}, 5)$. En effet, on a

$$
S(\mathcal{A}, 5)=|\mathcal{A}|-\left|\mathcal{A}_{2}\right|-\left|\mathcal{A}_{3}\right|+\left|\mathcal{A}_{6}\right|=\frac{1}{3} Y+\frac{2}{3}(\log 3) Z+r_{1}-r_{2}-r_{3}+r_{6}
$$

d'où $S(\mathcal{A}, 5)-W(5) Y=\frac{2}{3}(\log 3) Z+r_{1}-r_{2}-r_{3}+r_{6}$.

D'autre part, pour $y>1$ on pose

$$
\tau_{y}:=\frac{\log (x / \sqrt{y})}{\log y} .
$$

Ainsi, en appliquant le lemme 2.6 avec $q=z=p$ et $x / \sqrt{p}$ à la place de $x$, on a

$$
\begin{aligned}
S\left(\mathcal{A}_{p}, p\right) \leq & \frac{\omega(p)}{p} W(p)\left\{1+\mathrm{O}\left(\exp \left(-\tau_{p}\left(\log \tau_{p}+2\right)\right)\right)\right\} \\
& \times\left(Y-Z \log p+Z \sum_{p^{\prime}<p} \frac{\omega\left(p^{\prime}\right)}{p^{\prime}} \frac{\log p^{\prime}}{1-\omega\left(p^{\prime}\right) / p^{\prime}}\right)+\sum_{\substack{d \mid P(p) \\
d \leq x^{2} / p}} 3^{\Omega(d)}\left|r_{d p}\right|,
\end{aligned}
$$

d'où

$$
\begin{aligned}
& S\left(\mathcal{A}_{p}, p\right)-\frac{\omega(p)}{p} W(p) Y \\
& \leq \frac{\omega(p)}{p} W(p)\left[\left(\sum_{p^{\prime}<p} \frac{\omega\left(p^{\prime}\right)}{p^{\prime}} \frac{\log p^{\prime}}{1-\omega\left(p^{\prime}\right) / p^{\prime}}-\log p\right) Z\right. \\
& \quad+\mathrm{O}\left(\exp \left(-\tau_{p}\left(\log \tau_{p}+2\right)\right)\right) Y \\
& \left.\quad+\mathrm{O}\left(\exp \left(-\tau_{p}\left(\log \tau_{p}+2\right)\right)\left(\sum_{p^{\prime}<p} \frac{\omega\left(p^{\prime}\right)}{p^{\prime}} \frac{\log p^{\prime}}{1-\omega\left(p^{\prime}\right) / p^{\prime}}-\log p\right)\right) Z\right] \\
& +\sum_{d \mid P(p)} 3^{\Omega(d)}\left|r_{d p}\right| .
\end{aligned}
$$

Or, pour $z>5$ on a

$$
\begin{aligned}
& \sum_{5 \leq p<z}\left(-\frac{\omega(p)}{p} W(p) \log p+\left(\sum_{p^{\prime}<p} \frac{\omega\left(p^{\prime}\right)}{p^{\prime}} \frac{\log p^{\prime}}{1-\omega\left(p^{\prime}\right) / p^{\prime}}\right) \frac{\omega(p)}{p} W(p)\right) \\
(19)= & -\sum_{5 \leq p<z} \frac{\omega(p)}{p} W(p) \log p+\sum_{p^{\prime}<z} \frac{\omega\left(p^{\prime}\right)}{p^{\prime}} \frac{\log p^{\prime}}{1-\omega\left(p^{\prime}\right) / p^{\prime}} \sum_{p^{\prime}<p<z} \frac{\omega(p)}{p} W(p)
\end{aligned}
$$


car $p^{\prime}$ est implicitement $\geq 3$. Ainsi, par l'identité de Buchstab appliquée à $W$ (lemme 7.1, p. 204 de [5]), pour tout $p^{\prime}<z$ on a

$$
\begin{aligned}
\sum_{p^{\prime}<p<z} \frac{\omega(p)}{p} W(p) & =\sum_{p^{\prime \prime} \leq p<z} \frac{\omega(p)}{p} W(p)=W\left(p^{\prime \prime}\right)-W(z) \\
& =W\left(p^{\prime}\right)\left(1-\frac{\omega\left(p^{\prime}\right)}{p^{\prime}}\right)-W(z)
\end{aligned}
$$

avec $p^{\prime \prime}$ le plus petit nombre premier strictement supérieur à $p^{\prime}$, et par définition de $W$. Ceci donne, en remplaçant dans (19),

$$
\begin{aligned}
(18)= & -\sum_{5 \leq p<z} \frac{\omega(p)}{p} W(p) \log p \\
& +\sum_{p^{\prime}<z} \frac{\omega\left(p^{\prime}\right)}{p^{\prime}} \frac{\log p^{\prime}}{1-\omega\left(p^{\prime}\right) / p^{\prime}}\left(W\left(p^{\prime}\right)\left(1-\frac{\omega\left(p^{\prime}\right)}{p^{\prime}}\right)-W(z)\right) \\
= & \frac{2}{3} \log 3-W(z) \sum_{p^{\prime}<z} \frac{\omega\left(p^{\prime}\right)}{p^{\prime}} \frac{\log p^{\prime}}{1-\omega\left(p^{\prime}\right) / p^{\prime}} \\
= & \frac{2}{3} \log 3-W(z)(2 \log z+\mathrm{O}(1)) .
\end{aligned}
$$

De plus, par la formule de Mertens, on sait que pour $p<z$,

$$
\frac{W(p)}{W(z)}=\left(\frac{\log z}{\log p}\right)^{2}\left(1+\mathrm{O}\left(\frac{1}{\log p}\right)\right)
$$

ce qui implique

$$
\frac{W(p)}{W(z)} \ll\left(\frac{\log z}{\log p}\right)^{2}=\frac{\log p}{\log z}\left(\frac{\log z}{\log p}\right)^{3}
$$

d'où

$$
\begin{aligned}
& \sum_{p<z} \frac{\omega(p)}{p}(\log p) \exp \left(-\tau_{p}\left(\log \tau_{p}+2\right)\right.\left.+3 \log \left(\frac{\log z}{\log p}\right)\right) \\
& \ll(\log z) \exp (-\tau(\log \tau+1)),
\end{aligned}
$$

car par [5, p. 207-208], on a

$$
\exp \left(-\tau_{p}\left(\log \tau_{p}+2\right)+3 \log (\log z / \log p)\right) \leq \exp (-\tau(\log \tau+1)),
$$

ce qui majore la somme des (16), et de même

$$
\begin{aligned}
\sum_{p<z} \frac{\omega(p)}{p}(\log p)^{2} \exp \left(-\tau_{p}\left(\log \tau_{p}+2\right)+3 \log \left(\frac{\log z}{\log p}\right)\right) \\
\ll(\log z)^{2} \exp (-\tau(\log \tau+1)),
\end{aligned}
$$


ce qui majore la somme des (17). En outre, on a

$$
\left|r_{1}\right|+\left|r_{2}\right|+\left|r_{3}\right|+\left|r_{6}\right|+\sum_{5 \leq p<z} \sum_{\substack{d \mid P(p) \\ d \leq x^{2} / p}} 3^{\Omega(d)}\left|r_{d p}\right| \leq \sum_{\substack{d \mid P(z) \\ d \leq x^{2}}} 3^{\Omega(d)}\left|r_{d}\right| .
$$

Finalement, par la majoration (15) appliquée à (13), puis en utilisant les égalités (20) et (14) et la formule de Mertens, on a

$$
\begin{aligned}
S(\mathcal{A}, z) \geq & W(z)(Y+2 Z(\log z)(1+\mathrm{O}(1 / \log z))) \\
& +\mathrm{O}\left(\sum_{5 \leq p<z} \frac{\omega(p)}{p} W(p)(Y+Z \log p)\right) \\
& +r_{1}-r_{2}-r_{3}+r_{6}-\sum_{5 \leq p<z} \sum_{\substack{d \mid P(p) \\
d \leq x^{2} / p}} 3^{\Omega(d)}\left|r_{d p}\right| .
\end{aligned}
$$

Alors, en utilisant les majorations (21)-(23), on obtient

$$
\begin{aligned}
S(\mathcal{A}, z) \geq & W(z)\{1+\mathrm{O}(\exp (-\tau(\log \tau+1)))\}\{Y+2 Z(\log z)(1+\mathrm{O}(1 / \log z))\} \\
& -\sum_{\substack{d \mid P(z) \\
d \leq x^{2}}} 3^{\Omega(d)}\left|r_{d}\right| .
\end{aligned}
$$

En comparant avec la majoration du lemme 2.6, on conclut la preuve de la proposition 2.7 .

2.5. Minoration de $S(\mathcal{A}, z)$. Dans ce paragraphe, on donne une première minoration (théorème 2.8 ) de $S(\mathcal{A}, z)$, puis on en déduit le corollaire 2.11 qui sera utilisé dans la suite.

Pour obtenir une minoration, on itère une fois la majoration asymptotique (8) obtenue dans la proposition 2.3 :

THÉORÈme 2.8. Sous les hypothèses $(3)-(5)$, pour tout $\exp \left((\log x)^{6 / 7}\right) \leq$ $z \leq x^{2 / 5}$ tel que $Y-Z \log z \geq 0$ on a la minoration

$$
\begin{aligned}
S(\mathcal{A}, z) \geq & W(z)\left\{Y\left(1-\eta_{2}(2 \tau)\right)+Z(\log x) h_{1}(2 \tau)+Z(\log z)\left(2+h_{2}(2 \tau)\right)\right. \\
& \left.+\mathrm{O}\left(\left(Y+Z \frac{\log ^{2} z}{\log x}\right) \frac{\log _{2}^{8} x}{\log x}\right)\right\}-\sum_{\substack{d \mid P(z) \\
d \leq x^{2}}} 3^{\Omega(d)}\left|r_{d}\right|
\end{aligned}
$$

avec

$$
\tau:=\frac{\log x}{\log z}, \quad \eta_{2}(t):=2 t^{-2} \int_{t}^{\infty} u\left(\frac{1}{\sigma_{2}(u-1)}-1\right) d u
$$




$$
\begin{aligned}
& h_{1}(t):=2 t^{-2} \int_{t}^{\infty} \frac{u}{\sigma_{2}(u-1)}\left(\frac{3}{2} \frac{\sigma_{2}(u+3)}{\sigma_{2}(u-1)}-\frac{1}{2} \frac{\sigma_{2}(u+1)}{\sigma_{2}(u-1)}-1\right) d u, \\
& h_{2}(t):=t^{-1} \int_{t}^{\infty}\left[\frac{1}{\sigma_{2}(u-1)}\left(3-\frac{1}{2} \frac{\sigma_{2}(u+1)}{\sigma_{2}(u-1)}-\frac{9}{2} \frac{\sigma_{2}(u+3)}{\sigma_{2}(u-1)}\right)+2\right] d u .
\end{aligned}
$$

REMARQUE. Si l'on itère à nouveau le crible grâce à l'identité de Buchstab, on obtient une nouvelle majoration plus précise que (8). Mais cela amène de nombreux problèmes techniques (liés au calcul d'une intégrale double), et numériquement, il semble que l'on passe de 22.29 à 22.27 dans le théorème 1.5 (ce qui n'est pas très intéressant).

Preuve du théorème 2.8. On pose (comme dans le cas du crible de Selberg)

$$
z_{1}:=\exp \left(\frac{\log x}{\log _{2} x}\right)
$$

et on a ainsi $2 \leq z_{1} \leq z \leq x^{2 / 5}$. On a par l'identité de Buchstab (lemme 7.1, p. 204 de [5])

$$
S(\mathcal{A}, z)=S\left(\mathcal{A}, z_{1}\right)-\sum_{z_{1} \leq p<z} S\left(\mathcal{A}_{p}, p\right)
$$

Or, la proposition 2.7 donne

$$
\begin{aligned}
S\left(\mathcal{A}, z_{1}\right) \geq & W\left(z_{1}\right)\left\{1+\mathrm{O}\left(\exp \left(-\tau^{\prime}\left(\log \tau^{\prime}+1\right)\right)\right)\right\} \\
& \times\left\{Y+2 Z\left(\log z_{1}\right)\left(1+\mathrm{O}\left(1 / \log z_{1}\right)\right)\right\}-\sum_{\substack{d \mid P\left(z_{1}\right) \\
d \leq x^{2}}} 3^{\Omega(d)}\left|r_{d}\right|
\end{aligned}
$$

avec

$$
\tau^{\prime}:=\frac{\log x}{\log z_{1}}=\log _{2} x .
$$

Comme on a $([5$, p. 145])

$$
\frac{W\left(z_{1}\right)}{W(z)}\left\{1+\mathrm{O}\left(\exp \left(-\tau^{\prime}\left(\log \tau^{\prime}+1\right)\right)\right)\right\}=\left(\frac{\log z}{\log z_{1}}\right)^{2}+\mathrm{O}\left(\frac{\log _{2}^{3} x}{\log x}\right),
$$

on obtient finalement la minoration suivante à partir de (25) :

$$
\begin{aligned}
S\left(\mathcal{A}, z_{1}\right) \geq & W(z)\left\{\left(\frac{\log z}{\log z_{1}}\right)^{2}+\mathrm{O}\left(\frac{\log _{2}^{3} x}{\log x}\right)\right\}\left\{Y+2 Z \log z_{1}\right\} \\
& -\sum_{\substack{d \mid P\left(z_{1}\right) \\
d \leq x^{2}}} 3^{\Omega(d)}\left|r_{d}\right| .
\end{aligned}
$$

Pour traiter le deuxième terme du membre de droite de (24), on applique la proposition 2.3 avec $x / \sqrt{p}$ à la place de $x$ et $p$ à la place de $z$, en 
remarquant que

$$
1 / 2 \leq \tau-1 / 2 \leq \tau_{p} \leq \tau^{\prime}-1 / 2 \leq \log _{2} x
$$

avec

$$
\tau_{p}:=\frac{\log (x / \sqrt{p})}{\log p} .
$$

On obtient ainsi la majoration

$$
\begin{aligned}
& \sum_{z_{1} \leq p<z} S\left(\mathcal{A}_{p}, p\right) \\
& \leq \sum_{z_{1} \leq p<z} \frac{\omega(p)}{p} \frac{W(p)}{\sigma_{2}\left(2 \tau_{p}\right)}\{Y-Z \log p \\
& \quad+2 Z(\log p)\left(\frac{\tau_{p}}{2}+\frac{1+\tau_{p}}{4} \frac{\sigma_{2}\left(2 \tau_{p}+2\right)}{\sigma_{2}\left(2 \tau_{p}\right)}+\frac{3\left(1-\tau_{p}\right)}{4} \frac{\sigma_{2}\left(2 \tau_{p}+4\right)}{\sigma_{2}\left(2 \tau_{p}\right)}\right) \\
& \left.\quad+\mathrm{O}\left(\frac{\log _{2}^{5} x}{\log p}(Y-Z \log p+2 Z \log p)\right)\right\}+\sum_{z_{1} \leq p<z} \sum_{\substack{d \mid P(p) \\
d \leq x^{2} / p}} 3^{\Omega(d)}\left|r_{d p}\right|
\end{aligned}
$$

soit en développant $\tau_{p}$,

$$
\begin{aligned}
& \sum_{z_{1} \leq p<z} S\left(\mathcal{A}_{p}, p\right) \\
& \leq \sum_{z_{1} \leq p<z} \frac{\omega(p)}{p} \frac{W(p)}{\sigma_{2}\left(2 \tau_{p}\right)}\left\{Y+Z\left[\log x-\frac{3}{2} \log p\right.\right. \\
& \left.\quad+\frac{\sigma_{2}\left(2 \tau_{p}+2\right)}{\sigma_{2}\left(2 \tau_{p}\right)}\left(\frac{1}{4} \log p+\frac{1}{2} \log x\right)+\frac{\sigma_{2}\left(2 \tau_{p}+4\right)}{\sigma_{2}\left(2 \tau_{p}\right)}\left(\frac{9}{4} \log p-\frac{3}{2} \log x\right)\right] \\
& \left.\quad+\mathrm{O}\left(\frac{\log _{2}^{5} x}{\log p}(Y-Z \log p+2 Z \log p)\right)\right\}+\sum_{z_{1} \leq p<z} \sum_{\substack{d \mid P(p) \\
d \leq x^{2} / p}} 3^{\Omega(d)}\left|r_{d p}\right|
\end{aligned}
$$

Pour majorer chaque terme du membre de droite, on le compare à une intégrale. Pour cela, on rappelle d'abord le lemme 7.2, p. 214 de [5] (qui n'est qu'une combinaison de la sommation d'Abel et du théorème des nombres premiers) avec des hypothèses légèrement différentes :

LEMME 2.9. Soient $2 \leq z_{1} \leq z_{2} \leq z \leq x$. Si $\psi$ est une fonction positive, de classe $C^{1}$ pour $t \geq 1$ et telle qu'il existe $M>0$ pour lequel

$$
\sup _{t \geq 1}\left(|\psi(t)|+\left|\psi^{\prime}(t)\right|\right) \leq M
$$


alors

$$
\begin{aligned}
& \sum_{z_{1} \leq p<z_{2}} \frac{\omega(p)}{p} W(p) \psi\left(\frac{\log \left(x^{2} / p\right)}{\log p}\right) \\
& \quad=2 W(z)\left\{\left(\frac{\log z}{\log x^{2}}\right)^{2} \int_{\log x^{2} / \log z_{2}}^{\log x^{2} / \log z_{1}} t \psi(t-1) d t+\mathrm{O}_{M}\left(\frac{\log ^{2} z \log x}{\log ^{4} z_{1}}\right)\right\} .
\end{aligned}
$$

Preuve. Pour $z_{1} \leq w \leq z_{2}$ on pose

$$
\begin{aligned}
D(w) & :=\frac{1}{W(z)} \sum_{z_{1} \leq p<w} \frac{\omega(p)}{p} W(p) \\
& =\frac{W\left(z_{1}\right)}{W(z)}-\frac{W(w)}{W(z)}=\left(\log ^{2} z\right)\left(\frac{1}{\log ^{2} z_{1}}-\frac{1}{\log ^{2} w}+\mathrm{O}\left(\frac{1}{\log ^{3} z_{1}}\right)\right),
\end{aligned}
$$

et

$$
E(w):=\psi\left(\frac{\log \left(x^{2} / w\right)}{\log w}\right),
$$

qui est bien définie car $\log \left(x^{2} / w\right) / \log w \geq 1$. Alors, par intégration par parties, on a

$$
\begin{aligned}
& \frac{1}{W(z)} \sum_{z_{1} \leq p<z_{2}} \frac{\omega(p)}{p} W(p) E(p) \\
&=D\left(z_{2}\right) E\left(z_{2}\right)-\int_{z_{1}}^{z_{2}} D(w) d E(w) \\
&=\left(\log ^{2} z\right)\left(\frac{1}{\log ^{2} z_{1}}-\frac{1}{\log ^{2} z_{2}}\right) E\left(z_{2}\right)+\mathrm{O}_{M}\left(\frac{\log ^{2} z}{\log ^{3} z_{1}}\right) \\
&-\left(\log ^{2} z\right) \int_{z_{1}}^{z_{1}}\left(\frac{1}{\log ^{2} z_{1}}-\frac{1}{\log ^{2} w}\right) d E(w) \\
&+\mathrm{O}\left(\frac{\log ^{2} z \log ^{2}}{\log ^{3} z_{1}} \int_{z_{1}}^{z_{2}}\left|\psi^{\prime}\left(\frac{\log \left(x^{2} / w\right)}{\log w}\right)\right| \frac{d w}{w \log ^{2} w}\right) \\
&= 2\left(\log ^{2} z\right) \int_{z_{1}}^{z_{2}} \frac{E(w)}{\log ^{3} w} \frac{d w}{w}+\mathrm{O}_{M}\left(\frac{\log ^{2} z \log x}{\log ^{4} z_{1}}\right) .
\end{aligned}
$$

En faisant le changement de variable $w \mapsto\left(x^{2}\right)^{1 / t}$, l'intégrale devient

$$
\frac{1}{\log x^{2}} \int_{\log x^{2} / \log z_{2}}^{\log x^{2} / \log z_{1}} t \psi(t-1) d t
$$

d'où le lemme 2.9. 
Reprenons la preuve du théorème 2.8. On applique le lemme 7.2, p. 214 de [5] en prenant $\psi=1 / \sigma_{2}$, d'où

$$
\begin{aligned}
& \sum_{z_{1} \leq p<z} \frac{\omega(p)}{p} \frac{W(p)}{\sigma_{2}\left(2 \tau_{p}\right)} \\
& =W(z)\left(2\left(\frac{\log x^{2}}{\log z}\right)^{-2} \int_{\log x^{2} / \log z}^{\log x^{2} / \log z_{1}} \frac{t}{\sigma_{2}(t-1)} d t+\mathrm{O}\left(\frac{\log ^{2} z \log x}{\log ^{3} z_{1}}\right)\right) .
\end{aligned}
$$

Si on avait appliqué le lemme 2.9 directement (qui a des hypothèses plus faibles), on aurait obtenu $\log ^{4} z_{1}$ à la place de $\log ^{3} z_{1}$ dans le terme d'erreur, car dans la preuve du lemme 2.9 on est obligé de majorer trivialement $\left|\psi^{\prime}\right|$.

Or, on a

$$
\begin{aligned}
& \quad \int_{\log x^{2} / \log z}^{\log x^{2} / \log z_{1}} \frac{t}{\sigma_{2}(t-1)} d t \\
& \quad=\int_{\log x^{2} / \log z}^{\log x^{2} / \log z_{1}} t\left(\frac{1}{\sigma_{2}(t-1)}-1\right) d t+\frac{1}{2}\left(\frac{\log x^{2}}{\log z_{1}}\right)^{2}-\frac{1}{2}\left(\frac{\log x^{2}}{\log z}\right)^{2},
\end{aligned}
$$

d'où, par (26),

$$
\sum_{z_{1} \leq p<z} \frac{\omega(p)}{p} \frac{W(p)}{\sigma_{2}\left(2 \tau_{p}\right)} \leq W(z)\left\{\eta_{2}(2 \tau)+\left(\frac{\log z}{\log z_{1}}\right)^{2}-1+\mathrm{O}\left(\frac{\log _{2}^{3} x}{\log x}\right)\right\} .
$$

De la même façon, en prenant pour $\psi$ une des deux fonctions suivantes :

$$
t \mapsto \frac{\sigma_{2}(t+2)}{\sigma_{2}(t)^{2}}, \quad t \mapsto \frac{\sigma_{2}(t+4)}{\sigma_{2}(t)^{2}},
$$

par le lemme 2.9 on obtient

$$
\begin{aligned}
& \sum_{z_{1} \leq p<z} \frac{\omega(p)}{p} W(p) \frac{\sigma_{2}\left(2 \tau_{p}+2\right)}{\sigma_{2}\left(2 \tau_{p}\right)^{2}} \\
& =W(z)\left\{2(2 \tau)^{-2} \int_{2 \tau}^{\infty} u\left(\frac{\sigma_{2}(u+1)}{\sigma_{2}(u-1)^{2}}-1\right) d u+\left(\frac{\log z}{\log z_{1}}\right)^{2}-1+\mathrm{O}\left(\frac{\log _{2}^{4} x}{\log x}\right)\right\}
\end{aligned}
$$

et

$$
\begin{aligned}
& \sum_{z_{1} \leq p<z} \frac{\omega(p)}{p} W(p) \frac{\sigma_{2}\left(2 \tau_{p}+4\right)}{\sigma_{2}\left(2 \tau_{p}\right)^{2}} \\
&=W(z)\left\{2(2 \tau)^{-2} \int_{2 \tau}^{\infty} u\left(\frac{\sigma_{2}(u+3)}{\sigma_{2}(u-1)^{2}}-1\right) d u+\left(\frac{\log z}{\log z_{1}}\right)^{2}-1+\mathrm{O}\left(\frac{\log _{2}^{4} x}{\log x}\right)\right\} .
\end{aligned}
$$

Pour pouvoir appliquer le lemme 7.2 de [5] au lieu du lemme 2.9, il aurait fallu démontrer que chacune de ces fonctions $\psi$ est monotone, ce qui ne semble pas être le cas (elles le sont sans doute par morceaux). 
Pour démontrer le théorème 2.8 , on a aussi besoin du lemme suivant, qui est analogue au lemme 2.9 et dont nous omettrons la preuve :

Lemme 2.10. Soient $2 \leq z_{1} \leq z_{2} \leq z \leq x$. Si $\psi$ est une fonction positive, de classe $C^{1}$ pour $t \geq 1$ et telle qu'il existe $M>0$ pour lequel

$$
\sup _{t \geq 1}\left(|\psi(t)|+\left|\psi^{\prime}(t)\right|\right) \leq M
$$

alors

$$
\begin{aligned}
\sum_{z_{1} \leq p<z_{2}} & \frac{\omega(p)}{p} W(p)(\log p) \psi\left(\frac{\log \left(x^{2} / p\right)}{\log p}\right) \\
= & 2 W(z)\left\{\frac{\log ^{2} z}{\log x^{2}} \int_{\log x^{2} / \log z_{2}}^{\log z_{1}} \psi(t-1) d t+\mathrm{O}_{M}\left(\frac{\log ^{2} z \log x}{\log ^{3} z_{1}}\right)\right\} .
\end{aligned}
$$

On peut maintenant conclure la preuve du théorème 2.8. En appliquant le lemme 2.10 à $\psi=1 / \sigma_{2}$, on obtient

$$
\begin{aligned}
\sum_{z_{1} \leq p<z} \frac{\omega(p)}{p} & (\log p) \frac{W(p)}{\sigma_{2}\left(2 \tau_{p}\right)} \\
= & 2 W(z)\left\{\frac{\log ^{2} z}{\log x^{2}} \int_{\log x^{2} / \log z}^{\log x^{2} \log z_{1}} \frac{d t}{\sigma_{2}(t-1)}+\mathrm{O}\left(\frac{\log ^{2} z \log x}{\log ^{3} z_{1}}\right)\right\} \\
= & 2 W(z)\left\{\frac { \operatorname { l o g } ^ { 2 } z } { \operatorname { l o g } x ^ { 2 } } \left(\int_{\log x^{2} / \log z}^{\log x^{2} / \log z_{1}}\left(\frac{1}{\sigma_{2}(t-1)}-1\right) d t\right.\right. \\
& \left.\left.+\frac{\log x^{2}}{\log z_{1}}-\frac{\log x^{2}}{\log z}\right)+\mathrm{O}\left(\frac{\log ^{2} z \log x}{\log ^{3} z_{1}}\right)\right\} \\
= & 2 W(z)(\log z)\left((2 \tau)^{-1} \int_{2 \tau}^{\infty}\left(\frac{1}{\sigma_{2}(u-1)}-1\right) d u\right. \\
& \left.+\frac{\log z}{\log z_{1}}-1+\mathrm{O}\left(\frac{\log _{2}^{3} x}{\log ^{2} x} \log z\right)\right)
\end{aligned}
$$

De même, en l'appliquant aux fonctions

on a

$$
t \mapsto \frac{\sigma_{2}(t+2)}{\sigma_{2}(t)^{2}}, \quad t \mapsto \frac{\sigma_{2}(t+4)}{\sigma_{2}(t)^{2}}
$$

$$
\begin{aligned}
& \sum_{z_{1} \leq p<z} \frac{\omega(p)}{p}(\log p) W(p) \frac{\sigma_{2}\left(2 \tau_{p}+2\right)}{\sigma_{2}\left(2 \tau_{p}\right)^{2}} \\
& =2 W(z)(\log z)\left((2 \tau)^{-1} \int_{2 \tau}^{\infty}\left(\frac{\sigma_{2}(u+1)}{\sigma_{2}(u-1)^{2}}-1\right) d u+\frac{\log z}{\log z_{1}}-1+\mathrm{O}\left(\frac{\log _{2}^{3} x}{\log ^{2} x} \log z\right)\right)
\end{aligned}
$$


et

$$
\begin{aligned}
& \sum_{z_{1} \leq p<z} \frac{\omega(p)}{p}(\log p) W(p) \frac{\sigma_{2}\left(2 \tau_{p}+4\right)}{\sigma_{2}\left(2 \tau_{p}\right)^{2}} \\
= & 2 W(z) \log z\left((2 \tau)^{-1} \int_{2 \tau}^{\infty}\left(\frac{\sigma_{2}(u+3)}{\sigma_{2}(u-1)^{2}}-1\right) d u+\frac{\log z}{\log z_{1}}-1+\mathrm{O}\left(\frac{\log _{2}^{3} x}{\log ^{2} x} \log z\right)\right) .
\end{aligned}
$$

On a également par [4, p. 263], pour $z_{1} \leq p<z$,

$$
\frac{1}{\sigma_{2}\left(2 \tau_{p}\right)}=\mathrm{O}(1)
$$

et les majorations

$$
\begin{aligned}
\sum_{z_{1} \leq p<z} \frac{\omega(p)}{p \log p} \frac{W(p)}{\sigma_{2}\left(2 \tau_{p}\right)} \ll \sum_{z_{1} \leq p<z} \frac{\omega(p) W(p)}{p \log p} \ll W(z) \frac{\log _{2}^{4} x}{\log x}, \\
\sum_{z_{1} \leq p<z} \frac{\omega(p)}{p} \frac{W(p)}{\sigma_{2}\left(2 \tau_{p}\right)} \ll \sum_{z_{1} \leq p<z} \frac{\omega(p) W(p)}{p} \ll W(z) \frac{\log _{2}^{3} x}{\log ^{2} x} \log ^{2} z .
\end{aligned}
$$

En outre,

$$
\sum_{\substack{d \mid P\left(z_{1}\right) \\ d \leq x^{2}}} 3^{\Omega(d)}\left|r_{d}\right|+\sum_{z_{1} \leq p<z} \sum_{\substack{d \mid P(p) \\ d \leq x^{2} / p}} 3^{\Omega(d)}\left|r_{d p}\right| \leq \sum_{\substack{d \mid P(z) \\ d \leq x^{2}}} 3^{\Omega(d)}\left|r_{d}\right|
$$

d'où

$$
\begin{aligned}
S(\mathcal{A}, z) \geq & W(z)\left\{\frac{\log ^{2} z}{\log ^{2} z_{1}}\left(Y+2 Z \log z_{1}\right)+Y\left(1-\eta_{2}(2 \tau)-\frac{\log ^{2} z}{\log ^{2} z_{1}}\right)\right. \\
& +Z(\log x) h_{1}(2 \tau)+Z(\log z)\left(h_{2}(2 \tau)-2 \frac{\log z}{\log z_{1}}+2\right) \\
& \left.+\mathrm{O}\left(Y \frac{\log _{2}^{8} x}{\log x}\right)+\mathrm{O}\left(Z \frac{\log _{2}^{8} x}{\log ^{2} x} \log ^{2} z\right)\right\}-\sum_{\substack{d \mid P(z) \\
d \leq x^{2}}} 3^{\Omega(d)}\left|r_{d}\right|
\end{aligned}
$$

ce qui nous permet de conclure la preuve du théorème 2.8 avec les notations introduites dans l'énoncé.

On énonce maintenant un corollaire du théorème 2.8 qui traite le cas particulier dont nous aurons besoin par la suite.

Corollaire 2.11. Soient $A \in \mathbb{R}_{+}^{*}, B \in \mathbb{R}, X \geq 2$ et $0<\alpha<1,0<\beta$. On pose $Y=(A \log X+B) X, Z=A X$ et $x=X^{\alpha / 2} \log ^{-\beta} X$. Sous les hypothèses (3)-(5) avec ces valeurs de $Y$ et $Z$, sous l'hypothèse que $r_{d}$ vérifie

$$
\sum_{d<X^{\alpha} / \log ^{\beta} X} 3^{\Omega(d)}\left|r_{d}\right|=\mathrm{O}\left(\frac{X}{\log ^{3} X}\right)
$$


et pour tout $\exp \left(\log ^{6 / 7} x\right) \leq z \leq x^{2 / 5}$, il existe $X_{0}>0$ tel que l'on ait pour $X \geq X_{0}$ la minoration

$$
\begin{aligned}
S(\mathcal{A}, z) \geq & A W(z) X(\log X)\left\{\left(1-\eta_{2}(t)\right)\right. \\
& \left.+\frac{\alpha}{2} h_{1}(t)+\frac{\log z}{\log X}\left(2+h_{2}(t)\right)+\mathrm{O}\left(\frac{1}{\sqrt{\log X}}\right)\right\},
\end{aligned}
$$

avec

$$
t:=\alpha \frac{\log X}{\log z} .
$$

Preuve. On sait que (cf. [5, formule (6.4), p. 219])

$$
\eta_{2}(2 \tau)-\eta_{2}\left(\frac{\log X^{\alpha}}{\log z}\right) \ll \frac{\log _{2} X}{\log z} \frac{1}{\tau} \ll \frac{\log _{2} X}{\log X} .
$$

De même, on a

$$
\phi(2 \tau)-\phi\left(\frac{\log X^{\alpha}}{\log z}\right) \ll \frac{\log _{2} X}{\log z} \frac{1}{\tau} \ll \frac{\log _{2} X}{\log X}
$$

pour les fonctions $\phi$ suivantes :

$$
\begin{array}{ll}
t \mapsto 2 t^{-2} \int_{t}^{\infty} u\left(\frac{\sigma_{2}(u+1)}{\sigma_{2}(u-1)^{2}}-1\right) d u, \quad t \mapsto 2 t^{-2} \int_{t}^{\infty} u\left(\frac{\sigma_{2}(u+3)}{\sigma_{2}(u-1)^{2}}-1\right) d u, \\
t \mapsto t^{-1} \int_{t}^{\infty}\left(\frac{1}{\sigma_{2}(u-1)}-1\right) d u, \quad t \mapsto t^{-1} \int_{t}^{\infty}\left(\frac{\sigma_{2}(u+1)}{\sigma_{2}(u-1)^{2}}-1\right) d u, \\
t \mapsto t^{-1} \int_{t}^{\infty}\left(\frac{\sigma_{2}(u+3)}{\sigma_{2}(u-1)^{2}}-1\right) d u .
\end{array}
$$

Montrons-le par exemple pour la dernière fonction, i.e. pour

$$
\phi(t)=t^{-1} \int_{t}^{\infty}\left(\frac{\sigma_{2}(u+3)}{\sigma_{2}(u-1)^{2}}-1\right) d u .
$$

Soient $2<t_{1}<t_{2}$. On sait qu'il existe $\left.t_{3} \in\right] t_{1}, t_{2}[$ tel que

$$
\frac{\phi\left(t_{2}\right)-\phi\left(t_{1}\right)}{t_{2}-t_{1}}=\phi^{\prime}\left(t_{3}\right)
$$

et pour $t>1$ on a

$$
\phi^{\prime}(t)=-\frac{1}{t} \phi(t)-\frac{1}{t}\left(\frac{\sigma_{2}(t+3)}{\sigma_{2}(t-1)^{2}}-1\right) .
$$

Or, pour tout $u>2$,

$$
\frac{\sigma_{2}(u+3)}{\sigma_{2}(u-1)^{2}}-1 \geq 0
$$


car $\sigma_{2}$ est strictement croissante et $0<\sigma_{2}(u+3)<1$, donc

$$
\begin{aligned}
\sigma_{2}(u+3)- & \sigma_{2}(u-1)^{2} \\
& \geq \sigma_{2}(u+3)^{2}-\sigma_{2}(u-1)^{2} \\
& \geq\left(\sigma_{2}(u+3)-\sigma_{2}(u-1)\right)\left(\sigma_{2}(u+3)+\sigma_{2}(u-1)\right)>0 .
\end{aligned}
$$

Ainsi, on a

$$
0 \leq \phi\left(t_{3}\right)=t_{3}^{-1} \int_{t_{3}}^{\infty}\left(\frac{\sigma_{2}(u+3)}{\sigma_{2}(u-1)^{2}}-1\right) d u \leq \frac{1}{2} \int_{2}^{\infty}\left(\frac{\sigma_{2}(u+3)}{\sigma_{2}(u-1)^{2}}-1\right) d u=\phi(2)
$$

et

$$
0 \leq \frac{\sigma_{2}\left(t_{3}+3\right)}{\sigma_{2}\left(t_{3}-1\right)^{2}}-1 \leq \frac{1}{\sigma_{2}(1)^{2}}+1
$$

donc

$$
\phi^{\prime}\left(t_{3}\right) \ll \frac{1}{t_{3}},
$$

ce qui entraîne

$$
\phi\left(t_{2}\right)-\phi\left(t_{1}\right) \ll \frac{t_{2}-t_{1}}{t_{1}} .
$$

On a donc bien

$$
\phi(2 \tau)-\phi(t) \ll \frac{2 \tau-t}{2 \tau} \ll \frac{\log _{2} X}{\log z} \frac{1}{\tau} \ll \frac{\log _{2} X}{\log X} .
$$

On conclut enfin par invoquant le théorème 2.8. Le terme en $B$ rentre dans le $\mathrm{O}(1 / \sqrt{\log X})$.

3. Démonstration du théorème 1.5. Nous utiliserons les notations suivantes :

- $C_{\mathrm{c}}^{\infty}([a, b])$ désigne l'ensemble des fonctions $\mathbb{R} \rightarrow \mathbb{R}$, indéfiniment dérivables, à support compact inclus dans $[a, b]$.

- $g$ désigne une fonction appartenant à $C_{\mathrm{c}}^{\infty}([1,2])$.

- La transformée de Mellin d'une fonction $f: \mathbb{R}_{*}^{+} \rightarrow \mathbb{C}$, continue, bornée et nulle à l'infini, est définie pour $\Re s>0$ par

$$
\widehat{f}(s)=\int_{0}^{\infty} f(t) t^{s-1} d t .
$$

On applique le corollaire 2.11 aux deux suites $a_{n}^{+}$et $a_{n}^{-}$données par

$$
a_{n}^{ \pm}= \begin{cases}g(n / X)\left( \pm \mathrm{Kl}^{*}(1,1 ; n)+2^{\Omega(n)}\right) \geq 0 & \text { si } 2 \nmid n, \\ 0 & \text { sinon, }\end{cases}
$$

après avoir vérifié (§3.2) les hypothèses du crible étrange grâce à des résultats provenant de la théorie des formes automorphes. En comparant avec un 
équivalent de la somme

$$
\sum_{p \mid n \Rightarrow p \geq z} g(n / X) 2^{\Omega(n)}
$$

obtenu au lemme 3.2, on peut alors (§3.3) majorer

$$
\left|\sum_{p \mid n \Rightarrow p \geq z} g(n / X) \mathrm{Kl}^{*}(1,1 ; n)\right|
$$

avec une constante explicite dont on justifie le calcul numérique. On minore ensuite (§3.4) la quantité

$$
\sum_{p \mid n \Rightarrow p \geq z}\left|g(n / X) \mathrm{Kl}^{*}(1,1 ; n)\right|,
$$

en utilisant des résultats de géométrie algébrique. Cela permet (§3.5) de conclure la preuve du théorème 1.5.

3.1. Lemmes techniques. Démontrons d'abord un premier lemme, qui nous servira à vérifier (§3.2) les hypothèses du crible étrange.

Lemme 3.1. Pour tout d impair, $d \leq X$, on a l'égalité

$$
\begin{aligned}
\sum_{\substack{d \mid n \\
2 \nmid n}} 2^{\Omega(n)} g(n / X)= & \frac{2^{\Omega(d)}}{d} X\left\{\widehat{g}(1) G(1) \log X+c_{1}(g)-\widehat{g}(1) G(1) \log d\right\} \\
& +r^{\prime}(d, X)
\end{aligned}
$$

avec

$$
r^{\prime}(d, X):=\mathrm{O}_{g}\left(2^{\Omega(d)}(X / d)^{7 / 8}\right), \quad G(s):=\left(1-\frac{1}{2^{s}}\right)^{2} \prod_{p \geq 3}\left(1+\frac{1}{p^{s}\left(p^{s}-2\right)}\right),
$$

et $c_{1}(g)$ une constante ne dépendant que de $g$.

Preuve. On a par le théorème des résidus

$$
\begin{aligned}
\sum_{\substack{d \mid n \\
2 \nmid n}} 2^{\Omega(n)} g(n / X) & =\frac{1}{2 i \pi} \int_{2-i \infty}^{2+i \infty} \widehat{g}(s) F(s) X^{s} d s \\
& =\frac{1}{2 i \pi} \int_{7 / 8-i \infty}^{7 / 8+i \infty} \widehat{g}(s) \zeta^{2}(s) G(s) X^{s} d s+\operatorname{Res}_{s=1} \widehat{g}(s) \zeta^{2}(s) G(s) X^{s}
\end{aligned}
$$

avec

$$
F(s):=\prod_{p \geq 3}\left(1-\frac{2}{p^{s}}\right)^{-1}=\zeta^{2}(s) G(s)=\sum_{n \text { impair }} 2^{\Omega(n)} n^{-s} .
$$

Or,

$$
\operatorname{Res}_{s=1} \widehat{g}(s) \zeta^{2}(s) G(s) X^{s}=G(1) \widehat{g}(1) X \log X+c_{1}(g) X
$$


et

$$
\frac{1}{2 i \pi} \int_{7 / 8-i \infty}^{7 / 8+i \infty} \widehat{g}(s) \zeta^{2}(s) G(s) X^{s} d s=\mathrm{O}_{g}\left(X^{7 / 8}\right)
$$

donc

$$
\sum_{2 \nmid n} 2^{\Omega(n)} g(n / X)=\widehat{g}(1) G(1) X \log X+c_{1}(g) X+\mathrm{O}_{g}\left(X^{7 / 8}\right) .
$$

Ainsi, en appliquant (27) avec $X / d$ à la place de $X$, on obtient

$$
\begin{aligned}
\sum_{\substack{d \mid n \\
2 \nmid n}} 2^{\Omega(n)} g(n / X)= & 2^{\Omega(d)} \sum_{2 \nmid n} 2^{\Omega(n)} g(n d / X) \\
= & \frac{2^{\Omega(d)}}{d} X\left\{\widehat{g}(1) G(1) \log X+c_{1}(g)-\widehat{g}(1) G(1) \log d\right\} \\
& +r^{\prime}(d, X)
\end{aligned}
$$

où $r^{\prime}(d, X)=\mathrm{O}_{g}\left(2^{\Omega(d)}(X / d)^{7 / 8}\right)$. Ceci termine la preuve du lemme 3.1.

Nous utiliserons aussi le lemme suivant (cf. [2, proposition 4.2 et lemme 4.3]), qui donne un équivalent de

$$
\sum_{p \mid n \Rightarrow p \geq z} g(n / X) 2^{\Omega(n)}
$$

et nous permettra (par différence avec $\sum_{p \mid n \Rightarrow p \geq z} a_{n}^{ \pm}$) de majorer

$$
\left|\sum_{p \mid n \Rightarrow p \geq z} g(n / X) \mathrm{Kl}^{*}(1,1 ; n)\right|
$$

au $\S 3.3$.

Lemme 3.2. Soit $v_{0} \geq 2$. On a uniformément pour $2 \leq v \leq v_{0}$ et pour $z=X^{1 / v}$ la formule

$$
\sum_{\substack{n \leq 2 X \\ p \mid n \Rightarrow p \geq z}} 2^{\Omega(n)} g(n / X)=\widehat{g}(1) v(2 w(v)+(w * w)(v)) \frac{X}{\log X}+\mathrm{O}_{v_{0}, g}\left(\frac{X}{\log ^{2} X}\right),
$$

où w est la fonction de Buchstab définie sur $\mathbb{R}$ comme étant l'unique fonction continue pour $u \geq 1$ vérifiant

$$
\begin{cases}w(u)=0 & \text { pour } u<1 \\ u w(u)=1 & \text { pour } 1 \leq u \leq 2 \\ (u w(u))^{\prime}=w(u-1) & \text { pour } u>2\end{cases}
$$

et $*$ est la convolution de deux fonctions, définie par

$$
f * g(y)=\int_{-\infty}^{+\infty} f(x) g(y-x) d x
$$


En particulier, pour $v \geq 22$, il existe un réel $\theta,|\theta|<10^{-8}$ tel que

$$
\sum_{\substack{n \leq 2 X \\ p \mid n \Rightarrow p \geq z}} 2^{\Omega(n)} g(n / X)=\widehat{g}(1) e^{-2 \gamma}\left(v^{2}+2 v\right)(1+\theta) \frac{X}{\log X}+\mathrm{O}_{v_{0}, g}\left(\frac{X}{\log ^{2} X}\right) .
$$

3.2. Vérification des hypothèses du crible étrange. Dans ce paragraphe, on vérifie que l'hypothèse (4) du crible étrange est remplie par la suite $\left(a_{n}^{ \pm}\right)$, en montrant que la contribution des sommes de Kloosterman $\mathrm{Kl}^{*}(1,1 ; n)$ rentre dans le terme d'erreur $r_{d}$. Pour cela, on utilise la proposition suivante (proposition 2.1 de [2]) qui est issue de la théorie des formes automorphes.

Proposition 3.3. Pour tout $A>0$, il existe $B>0$ tel que l'on ait, pour $X \geq 1$, l'égalité

$$
\sum_{\substack{q \leq \sqrt{X} \log ^{-B} X \\ 2 \nmid q}}\left|\sum_{\substack{q \mid n \\ 2 \nmid n}} g\left(\frac{n}{X}\right) \frac{\mathrm{Kl}(1,1 ; n)}{\sqrt{n}}\right|=O_{A, g}\left(X \log ^{-A} 2 X\right) .
$$

On choisit les paramètres suivants, avec $B>0$ suffisamment grand :

$$
\begin{gathered}
\alpha:=\frac{1}{2}, \quad r_{d}:=r^{\prime}(d, X) \pm \sum_{\substack{d \mid n \\
2 \nmid n}} g(n / X) \mathrm{Kl}^{*}(1,1 ; n), \quad x:=X^{1 / 4} \log ^{-B} X, \\
Y:=\left(\widehat{g}(1) G(1) \log X+c_{1}(g)\right) X, \quad Z:=\widehat{g}(1) G(1) X .
\end{gathered}
$$

On remarque que les suites $\left(a_{n}^{ \pm}\right)$sont positives par le critère (1) d'EstermannWeil, et que

$$
\begin{aligned}
& \sum_{\substack{d \leq x^{2} \\
2 \nmid d}} 3^{\Omega(d)}\left|r_{d}\right| \\
& \ll \sum_{\substack{d \leq x^{2} \\
2 \nmid d}} 3^{\Omega(d)}\left|\sum_{\substack{d \mid n \\
2 \nmid n}} g(n / X) \mathrm{Kl}^{*}(1,1 ; n)\right|+\sum_{\substack{d \leq x^{2} \\
2 \nmid d}} 6^{\Omega(d)}(X / d)^{7 / 8} \\
& \left.\ll \underset{\substack{d \leq x^{2} \\
2 \nmid d}}{\ll} 9^{\Omega(d)}\left(\sum_{n \leq 2 X} 2^{\Omega(n)}\right)\right)^{1 / 2}\left(\sum_{\substack{d \leq x^{2} \\
2 \nmid d}}\left|\sum_{\substack{d \mid n \\
2 \nmid n}} g(n / X) \mathrm{Kl}^{*}(1,1 ; n)\right|\right)^{1 / 2}+X^{16 / 17}
\end{aligned}
$$

$\ll X / \log ^{3} X$

pour $B$ suffisamment grand, grâce à la proposition 3.3. On est donc bien dans l'hypothèse (4) du crible étrange, d'après le lemme 3.1. On peut donc appliquer le corollaire 2.11 aux suites $\left(a_{n}^{ \pm}\right)$.

3.3. Majoration de $\left|\sum_{n \leq 2 X, p \mid n \Rightarrow p \geq z} g(n / X) \mathrm{Kl}^{*}(1,1 ; n)\right|$. En prenant $v=$ 22.29 et $z=X^{1 / v}$, on trouve par calculs numériques en appliquant le corol- 
laire 2.11 ainsi que le lemme 3.2 que

$$
\left|\sum_{\substack{n \leq 2 X \\ p \mid n \Rightarrow p \geq z}} g(n / X) \mathrm{Kl}^{*}(1,1 ; n)\right| \leq 0.1565 \widehat{g}(1) \frac{X}{\log X}
$$

car

$$
S\left(\mathcal{A}^{ \pm}, z\right) \geq 170.5203 \widehat{g}(1) \frac{X}{\log X}
$$

et

$$
\sum_{\substack{n \leq 2 X \\ p \mid n \Rightarrow p \geq z}} g(n / X) 2^{\Omega(n)} \leq 170.6768 \widehat{g}(1) \frac{X}{\log X} .
$$

En effet, par définition de $\left(a_{n}^{ \pm}\right)$on a

$$
\pm \sum_{\substack{n \leq 2 X \\ p \mid n \Rightarrow p \geq z}} g(n / X) \mathrm{Kl}^{*}(1,1 ; n)=S\left(\mathcal{A}^{ \pm}, z\right)-\sum_{\substack{n \leq 2 X \\ p \mid n \Rightarrow p \geq z}} g(n / X) 2^{\Omega(n)}
$$

ce qui donne (28) en faisant la différence de (29) et (30) et en utilisant l'identité suivante (pour calculer (29)), conséquence de la formule de Mertens :

$$
\begin{aligned}
W(z) & =\prod_{3 \leq p<z}\left(1-\frac{2}{p}\right)=4 \prod_{p<z}\left(1-\frac{1}{p}\right)^{2} \prod_{3 \leq p<z} \frac{p^{2}-2 p}{(p-1)^{2}} \\
& =\frac{e^{-2 \gamma}}{\log ^{2} z} G(1)^{-1}\left(1+\mathrm{O}\left(\frac{1}{\log z}\right)\right) .
\end{aligned}
$$

Dans le reste de ce paragraphe, on va justifier les calculs numériques qui donnent la valeur 0.1565 dans (28). Ces calculs ont été effectués à l'aide de Mathematica en faisant d'abord une table de la fonction $\sigma_{2}$ puis en intégrant cette dernière par la méthode des rectangles sur un intervalle fini pour obtenir les fonctions $\eta_{2}$.

On peut en effet se limiter à un intervalle fini grâce au corollaire 3.5 cidessous, dont la preuve utilise le lemme suivant (qui est une version explicite du lemme 1, p. 263 de [4]) :

Lemme 3.4. Pour $s \geq 170$, on a la majoration

$$
1-\sigma_{2}(s) \leq 10^{-10} 85^{85}(s / 2)^{-s / 2},
$$

et pour $29 \leq s \leq 170$, on a la majoration

$$
1-\sigma_{2}(s) \leq 10^{-10}
$$

Preuve. On suit la démonstration de [4, p. 263] avec les mêmes notations, mais on va donner ici une majoration explicite du terme d'erreur. On pose 
$\sigma(s):=\sigma_{2}(2 s)$ et, pour $s>0$

$$
r(s):=\int_{0}^{\infty} e^{-s x} \exp \left(2 \int_{0}^{x} \frac{1-e^{-t}}{t} d t\right) d x .
$$

On remarque que $r$ est une fonction décroissante et qu'elle vérifie pour $s \geq 1$ les égalités

$$
s r(s)-2 \int_{s-1}^{s} r(x+1) d x=1, \quad s r(s) \sigma(s)-2 \int_{s-1}^{s} r(x+1) \sigma(x) d x=1 .
$$

Ainsi, si l'on pose $U(s):=1-\sigma(s) \geq 0$, on a pour $s \geq 1$ l'égalité

$$
s r(s) U(s)=2 \int_{s-1}^{s} r(x+1) U(x) d x \leq 2 r(s) \int_{s-1}^{s} U(x) d x,
$$

soit

$$
U(s) \leq \frac{2}{s} \int_{s-1}^{s} U(x) d x
$$

On pose, pour $s>0, \phi(s):=s \log s$ et $u(s):=U(s) \exp (\phi(s))$. En remarquant que pour $s-1 \leq x \leq s$,

$$
\phi(s)-\phi(x) \leq(s-x) \phi^{\prime}(s)
$$

on a la majoration

$$
\begin{aligned}
\frac{2}{s} \int_{s-1}^{s} U(x) d x & =\frac{2}{s} s^{-s} \int_{s-1}^{s} \exp (\phi(s)-\phi(x)) u(x) d x \\
& \leq \frac{2}{s} s^{-s} \int_{s-1}^{s} \exp \left((s-x) \phi^{\prime}(s)\right) d x \sup _{s-1 \leq x \leq s} u(x) \\
& \leq \frac{2}{s} s^{-s} \frac{\exp \left(\phi^{\prime}(s)\right)}{\phi^{\prime}(s)} \sup _{s-1 \leq x \leq s} u(x) .
\end{aligned}
$$

Or, pour $s \geq 85$,

$$
\frac{2}{s} \frac{\exp \left(\phi^{\prime}(s)\right)}{\phi^{\prime}(s)}=\frac{2 e}{\log s+1}<0.999
$$

d'où

$$
U(s) \leq 0.999 s^{-s} \sup _{s-1 \leq x \leq s} u(x) .
$$

Il nous reste donc à calculer $\sup _{s-1 \leq x \leq s} u(x)$. Par ce qui précède, pour $s \geq 85$ on a

$$
u(s) \leq 0.999 \sup _{s-1 \leq x \leq s} u(x) .
$$

On pose $A:=\max \left(1, \sup _{84 \leq x \leq 85} u(x)\right)$ et on va montrer par l'absurde que $u(s)$ est majorée par $A$ pour $s \geq 85$. Soit $s_{1}:=\inf \{s \mid s>85, u(s)>A\}$. 
S'il existe, $s_{1}$ est tel que $s_{1} \geq 85, u\left(s_{1}\right)=A$ et $u(s) \leq A$ pour $85 \leq s \leq s_{1}$ par continuité de $u$, et l'on a

$$
u\left(s_{1}\right) \leq 0.999 \sup _{s_{1}-1 \leq x \leq s_{1}} u(x),
$$

soit

$$
A \leq 0.999 A,
$$

ce qui est impossible car $A \geq 1$. Donc, finalement, $u(s) \leq A$ pour tout $s \geq 85$. On a donc montré que pour $s \geq 170$,

$$
1-\sigma_{2}(s) \leq A(s / 2)^{-s / 2} \text {. }
$$

D'autre part, par un calcul numérique, on trouve

$$
1-\sigma_{2}(29) \leq 10^{-10} \text {. }
$$

Comme $s \mapsto 1-\sigma_{2}(s)$ est décroissante, on en déduit le lemme 3.4.

On établit le corollaire suivant, qui justifie nos calculs numériques, car il permet d'intégrer la fonction $\sigma_{2}$ sur un intervalle fini :

Corollaire 3.5. Pour $2 \leq t \leq 30$, on a les majorations suivantes:

$$
\begin{array}{r}
\left|\eta_{2}(t)-2 t^{-2} \int_{t}^{30} u\left(\frac{1}{\sigma_{2}(u-1)}-1\right) d u\right| \leq \frac{4}{t^{2}} 10^{-6}, \\
\left|h_{1}(t)-2 t^{-2} \int_{t}^{30} \frac{u}{\sigma_{2}(u-1)}\left(\frac{3}{2} \frac{\sigma_{2}(u+3)}{\sigma_{2}(u-1)}-\frac{1}{2} \frac{\sigma_{2}(u+1)}{\sigma_{2}(u-1)}-1\right) d u\right| \leq \frac{8}{t^{2}} 10^{-6}, \\
\left|h_{2}(t)-t^{-1} \int_{t}^{30}\left[\frac{1}{\sigma_{2}(u-1)}\left(3-\frac{1}{2} \frac{\sigma_{2}(u+1)}{\sigma_{2}(u-1)}-\frac{9}{2} \frac{\sigma_{2}(u+3)}{\sigma_{2}(u-1)}\right)+2\right] d u\right| \\
\leq \frac{4}{t} 10^{-7} .
\end{array}
$$

Preuve. Par le lemme 3.4, la croissance de $\sigma_{2}$ et le fait que $\sigma_{2}(u) \leq 1$, on a

$$
\begin{aligned}
\int_{30}^{171} u\left(\frac{1}{\sigma_{2}(u-1)}-1\right) d u & \leq \frac{1}{\sigma_{2}(29)} \int_{29}^{170}\left(1-\sigma_{2}(u)\right) u d u \\
& \leq 10^{-10} \frac{1}{\sigma_{2}(29)} \int_{29}^{170} u d u \leq 1.5 \cdot 10^{-6}
\end{aligned}
$$

et encore, par le lemme 3.4 et la croissance de $\sigma_{2}$, 


$$
\begin{aligned}
\int_{171}^{\infty} u\left(\frac{1}{\sigma_{2}(u-1)}-1\right) d u & \leq \frac{1}{\sigma_{2}(170)} \int_{171}^{\infty}\left(1-\sigma_{2}(u-1)\right) u d u \\
& \leq \frac{1}{\sigma_{2}(170)} 10^{-10} 85^{85} \int_{171}^{\infty} u\left(\frac{u-1}{2}\right)^{-(u-1) / 2} d u \\
& \leq 2 \frac{1}{\sigma_{2}(170)} 10^{-10} 85^{85} \int_{85}^{\infty}(2 u+1) u^{-u} d u \\
& \leq 4.1 \cdot 10^{-10} 85^{85} \int_{85}^{\infty} u^{-u+1} d u
\end{aligned}
$$

Or, on remarque que

$$
\left(-v^{-v+1}\right)^{\prime}=v^{-v+1}(\log v+1-1 / v) \geq v^{-v+1},
$$

d'où

$$
\int_{171}^{\infty} u\left(\frac{1}{\sigma_{2}(u-1)}-1\right) d u \leq 6 \cdot 10^{-7}
$$

De même, on a

$$
\int_{30}^{\infty} \frac{u}{\sigma_{2}(u-1)}\left(\frac{\sigma_{2}(u+1)}{\sigma_{2}(u-1)}-1\right) d u \leq 2 \cdot 10^{-6}
$$

et

$$
\int_{30}^{\infty} \frac{u}{\sigma_{2}(u-1)}\left(\frac{\sigma_{2}(u+3)}{\sigma_{2}(u-1)}-1\right) d u \leq \frac{1}{\sigma_{2}(29)^{2}} \int_{30}^{\infty}\left(1-\sigma_{2}(u-1)\right) u d u \leq 2 \cdot 10^{-6} .
$$

De la même façon, par le lemme 3.4 et par la croissance de $\sigma_{2}$ on a

$$
\begin{aligned}
\int_{30}^{171}\left(\frac{1}{\sigma_{2}(u-1)}-1\right) d u & \leq \frac{1}{\sigma_{2}(29)} \int_{29}^{170}\left(1-\sigma_{2}(u)\right) d u \\
& \leq \frac{1}{\sigma_{2}(29)} 10^{-10} \int_{29}^{170} d u \leq 1.42 \cdot 10^{-8}
\end{aligned}
$$

et

$$
\begin{aligned}
\int_{171}^{\infty}\left(\frac{1}{\sigma_{2}(u-1)}-1\right) d u & \leq \frac{1}{\sigma_{2}(170)} \int_{171}^{\infty}\left(1-\sigma_{2}(u-1)\right) d u \\
& \leq \frac{1}{\sigma_{2}(170)} 10^{-10} 85^{85} \int_{171}^{\infty}\left(\frac{u-1}{2}\right)^{-(u-1) / 2} d u \\
& \leq 2.1 \cdot 10^{-10} 85^{85} \int_{85}^{\infty} u^{-u} d u
\end{aligned}
$$


Or, on remarque que

$$
\left(-v^{-v}\right)^{\prime}=v^{-v+1}(\log v+1) \geq v^{-v},
$$

d'où

$$
\int_{171}^{\infty}\left(\frac{1}{\sigma_{2}(u-1)}-1\right) d u \leq 3.2 \cdot 10^{-8}
$$

De même, on a

$$
\int_{30}^{\infty} \frac{1}{\sigma_{2}(u-1)}\left(\frac{\sigma_{2}(u+1)}{\sigma_{2}(u-1)}-1\right) d u \leq \frac{1}{\sigma_{2}(29)^{2}} \int_{29}^{\infty}\left(1-\sigma_{2}(u-1)\right) d u \leq 5 \cdot 10^{-8}
$$

et

$$
\int_{30}^{\infty} \frac{1}{\sigma_{2}(u-1)}\left(\frac{\sigma_{2}(u+3)}{\sigma_{2}(u-1)}-1\right) d u \leq \frac{1}{\sigma_{2}(29)^{2}} \int_{29}^{\infty}\left(1-\sigma_{2}(u-1)\right) d u \leq 5 \cdot 10^{-8},
$$

d'où le corollaire.

Remarque. Ce corollaire montre donc précisément que l'on peut tronquer toute intégrale sur un intervalle non borné de $\sigma_{2}(u)-1$ (ou de ses variantes). L'erreur ainsi commise est négligeable, ce qui est une conséquence logique du fait que $\sigma_{2}$ tend extrêmement vite vers 1 .

Ici, on prend $t=22.29 / 2$, donc l'erreur commise sera à chaque fois inférieure à $10^{-7}$, ce qui nous suffit pour faire nos calculs.

D'autre part, on rappelle que si $f \in C^{1}([a, b], \mathbb{R})$ avec $a<b$ alors pour tout $n \geq 1$ on a

$$
\left|\int_{a}^{b} f(t) d t-\frac{b-a}{n} \sum_{i=1}^{n} f\left(a+(b-a) \frac{i}{n}\right)\right| \leq \frac{(b-a)^{2}}{n} \sup _{t \in[a, b]}\left|f^{\prime}(t)\right| .
$$

Ceci permet de majorer l'erreur commise en utilisant la méthode des rectangles. Ici, on a pris $n$ de l'ordre de $2 \cdot 10^{6}$.

3.4. Minoration de $\sum_{n \leq 2 X, p \mid n \Rightarrow p \geq z}\left|g(n / X) \mathrm{Kl}^{*}(1,1 ; n)\right|$. Cette minoration repose sur la proposition suivante ([2, version effective de la proposition 5.2]) :

Proposition 3.6. Pour $v \geq 5$, il existe $X_{0}>0$ tel que pour $X \geq X_{0}$ et $z=X^{1 / v}$ on ait l'inégalité

$$
\sum_{\substack{n \leq 2 X \\ p \mid n \Rightarrow p \geq z}}\left|g(n / X) \mathrm{Kl}^{*}(1,1 ; n)\right| \geq c(v) \widehat{g}(1)(1-\mathrm{o}(1)) \frac{X}{\log X}
$$

avec

$$
c(v):=0.0006284 A_{3}(v)+0.001879 A_{4}(v)+0.000572 A_{5}(v),
$$


où

$$
\begin{aligned}
& A_{3}(v):=\int_{1 / v \max (x,(1-x) / 3)}^{1 / 3} \int_{1 / v}^{(1-x) / 2} \frac{d x d y}{x y(1-x-y)}, \\
& A_{4}(v):=\int_{x}^{1 / 4} \int_{\max (y,(1-x) / 3-y)}^{(1-x-y) / 2} \frac{d x d y d z}{x y z(1-x-y-z)}
\end{aligned}
$$

et

$$
A_{5}(v):=\int_{1 / v}^{1 / 6} \int_{x}^{(1-2 x) / 4} \int_{y}^{\min ((1-x-y) / 3,1 / 2-x-y)} \int_{\max (z,(1-x) / 3-y-z,(x+y+z) / 2)}^{(1-x-y-z) / 2} \frac{d x d y d z d t}{x y z t(1-x-y-z-t)} .
$$

La preuve de cette proposition est basée sur des résultats de géométrie algébrique, qui démontrent l'équidistribution des angles de certaines sommes d'exponentielles. On utilise ensuite un argument d'inclusion-exclusion, puis on conclut grâce au théorème des nombres premiers.

En calculant numériquement (à l'aide de Pari) les intégrales qui apparaissent dans la proposition 3.6, on obtient (avec $v=22.29$ ) la minoration explicite

$$
\sum_{\substack{n \leq 2 X \\ p \mid n \Rightarrow p \geq z}}\left|g(n / X) \mathrm{Kl}^{*}(1,1 ; n)\right| \geq 0.1568 \widehat{g}(1) \frac{X}{\log X}
$$

3.5. Fin de la démonstration. En comparant la majoration (28) obtenue au $\S 3.3$ et la minoration (31) obtenue au $\S 3.4$, on a

$$
\frac{\left|\sum_{\substack{n \leq 2 X \\ p \mid n \Rightarrow p \geq z}} g(n / X) \mathrm{Kl}^{*}(1,1 ; n)\right|}{\sum_{\substack{n \leq 2 X \\ p \mid n \Rightarrow p \geq z}}\left|g(n / X) \mathrm{Kl}^{*}(1,1 ; n)\right|} \leq \frac{0.1565}{0.1568}<1,
$$

ce qui démontre le théorème 1.5 .

\section{Références}

[1] T. Estermann, On Kloosterman's sum, Mathematika 8 (1961), 83-86.

[2] E. Fouvry et Ph. Michel, Sur le changement de signe des sommes de Kloosterman, Ann. of Math. 163 (2006), 1-40.

[3] - - - Crible asymptotique et sommes de Kloosterman, dans : Proceedings of the Session in Analytic Number Theory and Diophantine Equations, Bonner Math. Schriften 360, Univ. Bonn, 2003, 27 pp.

[4] G. Greaves, Sieves in Number Theory, Ergeb. Math. Grenzgeb. 43, Springer, 2001.

[5] H. Halberstam and H.-E. Richert, Sieve Methods, Academic Press, 1974. 
[6] H. Iwaniec and E. Kowalski, Analytic Number Theory, Amer. Math. Soc. Colloq. Publ. 53, Amer. Math. Soc., 2004.

[7] N. V. Kuznecov [N. V. Kuznetsov], The Petersson conjecture for cusp forms of weight zero and the Linnik conjecture. Sums of Kloosterman sums, Mat. Sb. (N.S.) 111 (153) (1980), 334-383 (in Russian); English transl.: Math. USSR-Sb. 39 (1981), 299-342.

[8] J. Sivak-Fischler, Crible asymptotique et sommes de Kloosterman, Bull. Soc. Math. France, à paraître.

Équipe d'Arithmétique et de Géométrie Algébrique

Université Paris-Sud

Bâtiment 425

91405 Orsay Cedex, France

E-mail: jimena.sivak@math.u-psud.fr 